HRJ

V.2 n.10 (2021)

Recebido: 04/01/2021

Aceito: 20/02/2021

\title{
Avaliação do perfil epidemiológico de pacientes com diagnóstico de Carcinoma Invasor de Colo Uterino
}

\author{
Bruna Gerolin Donaire ${ }^{1}$ \\ Sádia Martins de Paula Souza ${ }^{2}$ \\ Lucimara Priscila Campos Veras ${ }^{3}$ \\ Nádia Martins de Paula Souza ${ }^{4}$
}

${ }^{1}$ Médica residente em Ginecologia e Obstetrícia do Hospital Regional da Ceilândia, Distrito Federal, Brasil

2 Médica especialista em ginecologia oncológica, preceptora da residência de Ginecologia e Obstetrícia do Hospital Regional da Ceilândia, Distrito Federal, Brasil.

${ }^{3}$ Médica especialista em ginecologia, preceptora da residência de Ginecologia e Obstetrícia do Hospital Regional da Ceilândia, Distrito Federal, Brasil.

${ }^{4}$ Médica especialista em ginecologia, preceptora da residência de Ginecologia e Obstetrícia do Hospital Regional da Ceilândia, Distrito Federal, Brasil.

Endereço eletrônico para correspondência: bruh_donaire@hotmail.com

\section{RESUMO}

O câncer de colo uterino (CCU) representa o quarto tipo de câncer mais comum entre as mulheres no mundo. No Brasil, é a neoplasia maligna que mais acomete o trato genital feminino e são esperados 16.710 casos novos para 2021. Seu pico de incidência situa-se entre mulheres de 40 a 60 anos de idade. O principal agente promotor da lesão precursora é o Papilomavírus Humano (HPV), identificado em mais de 95\% dos casos. Além disso, fatores ligados à imunidade, à genética e ao comportamento sexual podem influenciar os mecanismos ainda incertos que determinam a regressão ou a progressão para lesões precursoras ou câncer. Considerando que o câncer de colo do útero é uma das prioridades da política de saúde pública do país, este trabalho avaliou o perfil epidemiológico das mulheres diagnosticadas com carcinoma invasor de colo uterino, atendidas na unidade de oncologia ginecológica de um hospital público e terciário, situado no Distrito Federal, utilizando como metodologia o estudo retrospectivo, quantitativo, transversal, descritivo e observacional. Foram identificados os principais fatores de risco, visando proporcionar diagnósticos precoces e tratamentos menos invasivos, além de estratégias de ação e intervenção mais eficazes a esta população.

Palavras-chave: Carcinoma. Colo Uterino. Perfil Epidemiológico 


\title{
Evaluation of the epidemiological profile of patientes diagnosed with Invasive Carcinoma of the Uterine Cervix
}

\begin{abstract}
Cervical cancer (CC) is the fourth most common cancer among women in the world. In Brazil, it is the malignant neoplasm that most affects the female genital tract, and 16.710 casos novos para 2021. Its peak incidence is between women 40 to 60 years of age. The main promoter of the precursor lesion is the Human Papillomavirus (HPV), identified in more than 95\% of cases. In addition, factors linked to immunity, genetics and sexual behavior seem to influence the still uncertain mechanisms that determine regression or progression to precursor lesions or cancer. Considering that cervical cancer is one of the priorities of public health policy in the country, this study evaluated the epidemiological profile of women diagnosed with invasive cervical carcinoma, attended at the gynecological oncology unit of a regional public hospital, located in the Federal District, using as methodology the retrospective, transversal, descriptive and observational study. The main risk factors were identified, aiming to provide early diagnoses and less invasive treatments, in addition to more effective action and intervention strategies for this population.
\end{abstract}

Keywords: Carcinoma. Uterine cervix. Epidemiological Profile

\section{INTRODUÇÃO}

O câncer do colo do útero, também denominado de câncer cervical, é uma doença de evolução gradativa, que se inicia com alterações neoplásicas intraepiteliais associadas, principalmente, aos chamados de tipos oncogênicos do papilomavírus humano - HPV. ${ }^{1}$ A exposição genital a este vírus é muito frequente e não causa doença na maioria das vezes, entretanto, em alguns casos, ocorrem transformações intraepiteliais progressivas que podem evoluir para um processo invasivo no colo uterino, em um período médio de 10 a 20 anos, que resulta em câncer. ${ }^{2}$

Este câncer apresenta etapas bem definidas, considerando que a doença demora em se desenvolver, por isso é possível a interrupção do seu curso a partir da prevenção correta, do diagnóstico precoce e do tratamento adequado 3 e, dentre todos os tipos de cânceres, é o que apresenta um dos mais altos potenciais de prevenção e cura. ${ }^{3,4}$

A neoplasia intraepitelial cervical (NIC) é uma longa fase de doença pré-invasiva, que precede o câncer de colo do útero. A NIC é categorizada em graus I, II e III, dependendo da 
proporção da espessura do epitélio que apresenta células maduras e diferenciadas. Os graus II e III têm maior probabilidade de progressão para o câncer, se não acompanhados ou não tratados. $^{5}$

O HPV aparece como condição necessária para o desenvolvimento, manutenção e progressão das lesões intraepiteliais, porém, faz-se necessária, além da persistência do vírus, sua associação com os outros fatores de risco como o tabagismo, multiplicidade de parceiros sexuais, multiparidade, uso de contraceptivos orais, baixa ingestão de vitaminas, início sexual precoce ou coinfecção por agentes infecciosos como o Vírus da Imunodeficiência Humana (HIV) e Chlamydia Trachomatis. ${ }^{6}$

O método de rastreamento do câncer do colo do útero e de suas lesões precursoras mais utilizado no Brasil é o exame citopatológico do colo do útero (Papanicolaou). ${ }^{2,3,4} \mathrm{~A}$ coleta deve ser iniciada aos 25 anos de idade em mulheres sexualmente ativas, sendo que os dois primeiros exames devem ser realizados com intervalo anual e, se ambos os resultados forem negativos, os próximos devem ser realizados a cada 3 anos, até os 64 anos. Seu objetivo é detectar células negativas ou positivas para neoplasia intraepitelial ou malignidade na ectocérvice e endocérvice de mulheres com colo aparentemente normal. ${ }^{4,7}$

Existem várias nomenclaturas para classificação dos resultados citológicos, entretanto, a mais usada para classificação dos resultados citológicos é o sistema Bethesda, atualizada em 2014, que padroniza o conceito de lesões intraepiteliais de baixo grau (LSIL) e lesões intraepiteliais de alto grau (HSIL) $)^{7,8}$.

As Diretrizes Brasileiras para o Rastreamento do Câncer do Colo do Útero 3 designam que quando há alteração citológica deve ser feita a investigação colposcópica.,3,7 Por enquanto, não é recomendado o rastreamento do DNA do HPV, mas este método vem sendo utilizado progressivamente no mundo, contudo, a análise histopatológica através de biópsia guiada por colposcopia é o padrão ouro para o diagnóstico. ${ }^{7}$ 
As manifestações clínicas do câncer de colo uterino podem ser assintomáticas, quando em fases iniciais ou estarem associadas a sangramento vaginal anormal, corrimento vaginal serossanguíneo e até dor pélvica com edema de membros inferiores e hidronefrose por doença avançada. As opções de tratamento incluem cirurgia, radioterapia e quimioterapia, e podem ser usadas em combinação. ${ }^{9} \mathrm{O}$ tratamento do câncer cervical depende do estadiamento clínico do tumor, de comorbidades associadas, da idade e do estado geral da paciente, além do seu desejo de gestar e de condições disponíveis no serviço de saúde. ${ }^{10}$

Em 2006, a Agência Nacional de Vigilância Sanitária (ANVISA) regulamentou a comercialização da vacina que previne contra infecções provocadas por alguns tipos do vírus HPV. A vacina, disponível pelo Programa Nacional de Imunização do Ministério da Saúde, é a quadrivalente, oferecendo imunidade contra os subtipos $6,11,16,18$, prevenindo contra o câncer cervical (colo uterino), vulvar, anal, além da verruga genital, tendo como público-alvo meninas entre 9 a 14 anos e meninos entre 11 a 14 anos, disponível também para pessoas de 9 a 26 anos com HIV, em transplantados e em tratamento oncológico. ${ }^{12,13,14}$

A estimativa mundial indica que o câncer cervical foi o quarto mais frequente em todo o mundo, ${ }^{2,14}$ com ocorrência de 570 mil casos novos diagnosticados em 2018 e mais de 311 mil mortes. Estima-se que, em 2030, esta neoplasia seja responsável pela morte de 474 mil mulheres. Portanto, considera-se um problema de saúde pública mundial, por exercer papel importante na morbimortalidade das mulheres, além de ser um desafio às políticas públicas, principalmente em países de média e baixa renda. ${ }^{11}$ De acordo com o Instituto Nacional do Câncer (INCA)², no Brasil, é o terceiro tumor maligno mais frequente na população feminina, superado apenas pelo câncer mamário e colorretal, e a quarta causa de morte de mulheres por câncer. O número de mortes pelo câncer de colo de útero em 2019 foi de 6.596 óbitos, ${ }^{2}$ sendo que, para cada ano do triênio 2020-2022, estima-se um total de 16.710 , com risco estimado de 16,35 casos a cada 100 mil mulheres. ${ }^{16}$ 
Segundo Gonçalves et al, ${ }^{14}$ a problematização da doença se deve ao reduzido acesso aos serviços de saúde, aos programas de vacinação, de rastreio e tratamento precoce, pois a recomendação da Organização Mundial de Saúde (OMS) é que a cobertura atinja uma população de pelo menos $80 \%$, garantindo acesso ao diagnóstico e ao tratamento adequado para que haja uma redução de 60 a $90 \%$ na incidência de CCU, impactando diretamente na redução da morbimortalidade., 3

Diante do exposto, o objetivo deste estudo foi avaliar o perfil epidemiológico das mulheres diagnosticadas com carcinoma invasor de colo uterino visando identificar os principais fatores de risco, para possibilitar diagnósticos precoces e tratamentos menos invasivos, sugerindo estratégias de ação e intervenção mais eficazes a esta população.

\section{MÉTODO}

Trata-se de um estudo retrospectivo, quantitativo, transversal, descritivo e observacional, que se propôs a avaliar o perfil epidemiológico de pacientes diagnosticadas com carcinoma invasor de colo uterino, atendidas na Unidade de Oncologia Ginecológica de um hospital público e terciário, situado no Distrito Federal, entre janeiro de 2016 a dezembro de 2019. Foi aplicada análise estatística descritiva, de acordo com a natureza das variáveis, sendo informados os valores absolutos (n) e percentuais (\%) dos dados analisados e demonstrados em tabelas.

\section{COLETA DE DADOS}

As pacientes incluídas no estudo foram selecionadas a partir dos seguintes critérios: maiores de 18 anos, com diagnóstico de carcinoma invasor de colo uterino entre janeiro de 2016 e dezembro de 2019, derivados de biópsias de fragmento, conização a Scott ou conização por cirurgia de alta frequência (CAF), provenientes do serviço de anatomia 
patológica do Hospital Regional de Ceilândia (HRC). Os dados foram coletados através de um relatório, cujas informações foram obtidas do prontuário eletrônico (TrakCare ${ }^{\circledR}$ ) da Secretaria de Estado de Saúde do Distrito Federal (SES-DF), sendo descartados os casos de informações essenciais incompletas, biópsias provenientes de outros serviços de saúde, laudos obtidos através de histerectomias e laudo cujo diagnóstico histopatológico foi de cervicite crônica, neoplasia intraepitelial e carcinoma in situ.

Os dados foram tabulados no software Microsoft Office Excel®, onde foram analisadas as seguintes variáveis: idade (dado obrigatório), estado civil, menarca, sexarca, número de parceiros sexuais, paridade, tabagismo, método contraceptivo, motivo do encaminhamento ao setor, realização ou não de exame preventivo nos últimos 3 anos, resultado do último exame de citologia oncótica (preventivo), colposcopia, tipo histológico, estadiamento clínico, tratamento proposto e desfecho.

Por se tratar de busca ativa no registro eletrônico dos resultados histopatológicos de colo uterino no Setor de Patologia do HRC e da análise dos prontuários eletrônicos das pacientes, é sabido se tratar de informações sigilosas, portanto, foi realizada cuidadosa análise a fim de evitar o risco de exposição dos seus dados.

\section{SISTEMATIZAÇÃO DO TRABALHO}

Foram identificados os resultados histopatológicos de colo uterino, segregados por ano, e selecionados os que continham diagnóstico de carcinoma invasor de colo uterino para avaliação. Após a identificação do diagnóstico, foi realizada a avaliação do prontuário eletrônico destas pacientes e coletados seus dados clínicos e epidemiológicos para análise.

Inicialmente, foram coletados os dados de 100 pacientes, das quais 3 foram excluídas por não apresentarem dados essenciais. Portanto, o total bruto, cujos dados foram analisados, 
foi de 97 pacientes, sendo analisados de forma estratificada os dados de formulários completos em relação às outras variáveis selecionadas.

\section{RESULTADOS E DISCUSSÃO}

A análise quanto ao estado civil mostrou que 67\% (65 pacientes) referiam estabilidade conjugal, enquanto apenas 19\% (18 pacientes) informaram ser solteiras e 14\% (14 pacientes) não tinham este registro. Com relação ao método contraceptivo utilizado, 20\% (19 pacientes) utilizavam o anticoncepcional, 16\% (16 pacientes) já fizeram laqueadura, 30\% (29 pacientes)

já estavam na menopausa, apenas 3\% (3 pacientes) utilizavam preservativo, $13 \%$ (13 pacientes) não utilizavam nenhum método e $18 \%$ dos registros não foram informados. Quanto ao tabagismo, 64\% (62 pacientes) informaram não ser fumantes, contra $28 \%$ (27 pacientes) fumantes e $8 \%$ ( 8 pacientes) não tinham dados registrados.

O encaminhamento ao setor ocorreu, principalmente, por sangramento uterino anormal (SUA) acometido por 45\% (44 pacientes), seguido de 30\% (29 pacientes) com alteração no exame de citologia oncótica (CCO), 20\% (19 pacientes) com alterações no exame físico e 5\% (5 pacientes) com SUA e alterações no exame físico. Os resultados do último CCO realizado apresentaram 19\% (18 pacientes) com HSIL, 7\% (7 pacientes) com células escamosas atípicas, não se podendo descartar uma lesão de alto grau (ASCH), 6\% (6 pacientes) com carcinoma epidermóide invasor, 12\% (12 pacientes) apresentaram resultado normal, 47\% (46 pacientes) não realizaram o exame e $8 \%$ dos registros não tinham esses dados. Nos resultados da colposcopia realizada, $21 \%$ (20 pacientes) dos resultados foram não indicativos de invasão, $77 \%$ (75 pacientes) foram indicativos de invasão e $2 \%$ dos casos não continham dados registrados.

Na Tabela 1, a seguir, verificou-se que a média das idades das pacientes foi de 49 anos, o que corrobora com o INCA, ${ }^{17}$ cujas estatísticas apontam que o câncer do colo do útero 
é raro em mulheres até 30 anos e o auge de sua incidência se dá na faixa etária de 45 a 50 anos. A menarca teve como média os 13 anos, porém 24 pacientes não tinham dados registrados e a média de idade da sexarca seria aos 17 anos, mas faltam dados de 32 pacientes, por isso ambos os critérios não puderam ser avaliados devido à alta taxa de falta de informações.

Tabela 1. Características epidemiológicas de mulheres com carcinoma invasor de colo uterino em relação à idade atual e menarca das pacientes atendidas, Distrito Federal, 20162019.

\begin{tabular}{c|c|c}
\hline \multicolumn{1}{c|}{ Características } & $\mathrm{n}$ & Desvio padrão \\
\hline Média das idades, em anos & 49 & \pm 15 \\
\hline
\end{tabular}

Fonte: Ficha Padrão para o Estudo

Os tipos histológicos foram analisados e comparados com a média prevalente das idades em cada um e foram identificados (Tabela 2) que a maioria dos casos apresentou o Carcinoma epidermóide (78\%), cuja média prevalente das idades foi de 50 anos, seguido do Adenocarcinoma (13\% - 48 anos), Carcinoma epidermóide microinvasor (6\% - 40 anos) e Carcinoma epidermóide com adenocarcinoma (2\% - 56 anos). Estes dados corroboram com os encontrados na literatura. ${ }^{11}$ 
Tabela 2. Distribuição, conforme tipo histopatológico, do total das pacientes atendidas, com identificação da média prevalente das idades para cada tipo, Distrito Federal, 2016-2019.

\begin{tabular}{l|c|c|c}
\hline \multicolumn{1}{c|}{ Tipos Histológicos } & $\mathrm{n}$ & $\%$ & $\begin{array}{c}\text { Média prevalente } \\
\text { das idades }\end{array}$ \\
\hline Adenocarcinoma & 13 & 13 & 48 \\
Carcinoma epidermóide & 76 & 78 & 50 \\
Carcinoma epidermóide com adenocarcinoma & 2 & 2 & 56 \\
Carcinoma epidermóide microinvasor & 6 & 6 & 40 \\
\hline
\end{tabular}

Fonte: Ficha Padrão para o Estudo

Outra comparação realizada foi entre o estadiamento clínico e os tipos histológicos prevalentes (Tabela 3). Encontrou-se maioria dos casos com estádio IIB (27\%), cuja predominância histológica ocorreu no tipo Carcinoma epidermóide (26\%), entretanto, foram verificados casos deste tipo em todos os estádios analisados. Outros estádios que se destacaram foram o IVA (18\%) e IIIB (12\%), que também tiveram predominância no tipo Carcinoma epidermóide (13\% e 10\% respectivamente). No tipo histológico Adenocarcinoma, as ocorrências apresentaram prevalência no estádio IVA (3\%), com uma pequena diferença em relação aos demais: IA1 (1\%), IB1 (2\%), IB2 (1\%), IIB (1\%), IIIA (2\%), IIIB (2\%) e IIIC (1\%).

A porcentagem de pacientes diagnosticadas com o Adenocarcinoma pode ser considerada acima da média brasileira, que, de acordo com Brasil ${ }^{3}$, foi de $0,01 \%$ em 2013, por exemplo. Apesar da baixa incidência dos demais tipos, os estádios, que foram encontrados, demonstram alto grau de extensão nas amostras observadas. 
Tabela 3. Distribuição, conforme estadiamento clínico, do total das pacientes atendidas com identificação do tipo histológico para cada caso, Distrito Federal, 2016-2019

\begin{tabular}{|c|c|c|c|c|c|c|}
\hline \multirow[b]{2}{*}{$\begin{array}{l}\text { Estadiamento } \\
\text { Clínico }\end{array}$} & \multirow[b]{2}{*}{$\mathrm{n}$} & \multirow[b]{2}{*}{$\%$} & \multicolumn{4}{|c|}{ Tipo Histológico prevalente } \\
\hline & & & Adenocarcinoma & $\begin{array}{l}\text { Carcinoma } \\
\text { epidermóide }\end{array}$ & $\begin{array}{c}\text { Carcinoma } \\
\text { epidermóide } \\
\text { com adenocarcinoma }\end{array}$ & $\begin{array}{l}\text { Carcinoma } \\
\text { epidermóide } \\
\text { microinvasor }\end{array}$ \\
\hline IA1 & 4 & 4 & $1 \%$ & $4 \%$ & - & - \\
\hline IA2 & 2 & 2 & - & $1 \%$ & - & $1 \%$ \\
\hline IB1 & 6 & 6 & $2 \%$ & $4 \%$ & - & - \\
\hline IB2 & 6 & 6 & $1 \%$ & $5 \%$ & - & - \\
\hline IIA1 & 4 & 4 & - & $3 \%$ & - & $1 \%$ \\
\hline IIA2 & 3 & 3 & - & $2 \%$ & $1 \%$ & - \\
\hline IIB & 26 & 27 & $1 \%$ & $26 \%$ & - & - \\
\hline IIIA & 8 & 8 & $2 \%$ & $5 \%$ & - & $1 \%$ \\
\hline IIIB & 12 & 12 & $2 \%$ & $10 \%$ & - & - \\
\hline IIIC & 5 & 5 & $1 \%$ & $3 \%$ & - & $1 \%$ \\
\hline IVA & 17 & 18 & $3 \%$ & $13 \%$ & - & $1 \%$ \\
\hline IVB & 2 & 2 & - & $1 \%$ & $1 \%$ & - \\
\hline
\end{tabular}

Fonte: Ficha Padrão para o Estudo

Foi possível, também, verificar a relação entre o nível de estadiamento clínico e a colpocitologia oncótica (CCO) (Tabela 4), em que se observou a quantidade de pacientes que 
realizaram ou não o exame de CCO nos últimos 3 anos, conforme recomendação. ${ }^{3}$ A maioria das pacientes que não fez o exame (47\%), apresentou os estádios mais avançados: IIB (15\%), IIIA (7\%), IIIB (4\%), IIIC (3\%), IVA (12\%) e IVB (1\%); apenas uma destas paciente apresentou o estádio IA1, considerado microinvasivo. ${ }^{11}$ Dentre as que fizeram o exame, também houve casos de estádios avançados consideráveis: IIB (11\%), IIIB (8\%) e IVA (5\%).

Tabela 4. Quantidade de pacientes verificada na relação entre o nível de estadiamento clínico e a realização do exame de CCO nos últimos 3 anos, Distrito Federal, 2016-2019

\begin{tabular}{|c|c|c|}
\hline \multirow{2}{*}{ Estadiamento Clínico } & \multicolumn{2}{|c|}{ Realizou exame de CCO nos últimos 3 anos } \\
\hline & Sim & Não \\
\hline IA 1 & 4 & 1 \\
\hline IA 2 & 2 & 0 \\
\hline IB 1 & 6 & 0 \\
\hline IB2 & 6 & 0 \\
\hline IIA1 & 2 & 2 \\
\hline IIA2 & 1 & 2 \\
\hline IIB & 11 & 15 \\
\hline IIIA & 1 & 7 \\
\hline IIIB & 8 & 4 \\
\hline IIIC & 2 & 3 \\
\hline
\end{tabular}




\begin{tabular}{c|c|c} 
IVA & 5 & 12 \\
IVB & 1 & 1 \\
& Fonte: Ficha Padrão para o Estudo
\end{tabular}

Em relação aos tratamentos administrados, a correlação com a idade, estadiamento clínico e desfecho (Tabelas 5, 6 e 7) demonstraram que a quimiorradioterapia (80\%) supera os demais tratamentos, conforme esperado, pois os estadiamentos clínicos predominantes foram o IIB em diante (72\%), que, segundo a classificação da Federação Internacional de Ginecologia e Obstetrícia (FIGO), devem ser tratados com Quimioterapia (cisplatina com ou sem 5-fluoracil) e Radioterapia simultaneamente, o que aumenta a sobrevida global em $12 \%$ e livre de doença em 16\%. As ocorrências em todos os estádios mais avançados, sem possibilidade de abordagem cirúrgica, podem explicar o índice de desfecho por óbito (23\%). ${ }^{11}$

A média das idades das 5 pacientes que não realizaram tratamento foi de 54 anos e, para todas, o desfecho foi o óbito, o que demonstra a seriedade com que devem ser realizadas as ações de prevenção, diagnóstico e tratamento precoce. 
Tabela 5. Relação entre os tratamentos administrados e a média prevalente das idades do total de pacientes atendidas, Distrito Federal, 2016-2019.

\begin{tabular}{l|c|c|c}
\multicolumn{1}{c|}{ Tratamento } & $\mathrm{n}$ & $\%$ & $\begin{array}{c}\text { Média prevalente } \\
\text { das idades }\end{array}$ \\
\hline Quimiorradioterapia & 76 & 78 & 50 \\
Conização & 5 & 5 & 43 \\
Wertheim Meigs & 5 & 5 & 43 \\
Wertheim Meig + Quimiorradioterapia & 4 & 4 & 41 \\
Não realizou & 5 & 5 & 54 \\
Sem dado registrado & 2 & 2 & - \\
\hline
\end{tabular}

Fonte: Ficha Padrão para o Estudo

Tabela 6. Relação entre estadiamento clínico e os tratamentos aplicados ao total de pacientes atendidas (2 pacientes não apresentaram registro de tratamento - 2\%), Distrito Federal, 20162019.

\begin{tabular}{c|c|c|c|c|c}
\hline $\begin{array}{c}\text { Estadiamento } \\
\text { Clínico }\end{array}$ & Quimiorradioterapia & Conização & $\begin{array}{c}\text { Wertheim } \\
\text { Meigs }\end{array}$ & $\begin{array}{c}\text { Wertheim Meigs + } \\
\text { Quimiorradioterapia }\end{array}$ & $\begin{array}{c}\text { Não } \\
\text { realizou }\end{array}$ \\
\hline IA1 & - & $4 \%$ & $1 \%$ & - & - \\
IA2 & - & - & $1 \%$ & - & - \\
IB1 & $1 \%$ & - & $2 \%$ & $3 \%$ & - \\
IB2 & $5 \%$ & - & $1 \%$ & - & - \\
IIA1 & $4 \%$ & - & - &
\end{tabular}




\begin{tabular}{|c|c|c|c|c|c|}
\hline IIA2 & $2 \%$ & - & - & $1 \%$ & - \\
\hline IIB & $25 \%$ & - & - & - & $1 \%$ \\
\hline IIIA & $8 \%$ & - & - & - & - \\
\hline IIIB & $13 \%$ & - & - & - & - \\
\hline IIIC & $5 \%$ & - & - & - & - \\
\hline IVA & $15 \%$ & - & - & - & $3 \%$ \\
\hline IVB & $1 \%$ & - & - & - & $1 \%$ \\
\hline
\end{tabular}

Fonte: Ficha Padrão para o Estudo

Tabela 7. Relação entre tratamento aplicado e desfecho do total de pacientes $(n=95)$ atendidas, Distrito Federal, 2016-2019.

\begin{tabular}{l|c|c|c|c}
\hline \multicolumn{1}{c|}{ Tratamento } & $\begin{array}{c}\text { Seguimento } \\
\text { até 2 anos }\end{array}$ & $\begin{array}{c}\text { Seguimento até } \\
2 \text { anos a 5 anos }\end{array}$ & $\begin{array}{c}\text { Perda de } \\
\text { seguimento }\end{array}$ & Óbito \\
\hline Quimiorradioterapia (QR) & $27 \%$ & $12 \%$ & $22 \%$ & $19 \%$ \\
Conização & - & - & - & - \\
Wertheim Meigs & $2 \%$ & $2 \%$ & - & - \\
Wertheim Meigs +QR & - & - & - & $5 \%$ \\
Não realizou & - & - & $2 \%$ & - \\
\hline
\end{tabular}

Fonte: Ficha Padrão para o Estudo 


\section{CONCLUSÕES}

O câncer do colo uterino se apresenta como importante problema de saúde pública mundial. Entre todas as neoplasias, a do colo uterino tem um dos mais altos potenciais de prevenção e cura, além de bom prognóstico quando diagnosticada precocemente, porém, quando tardio pode ser o principal responsável pela manutenção das relevantes taxas de mortalidade.

O trabalho demonstrou que a maioria das pacientes foi diagnosticada com estádio mais avançado, o que indicou tratamentos mais agressivos e alta taxa de mortalidade. Estes resultados também conduzem à reflexão sobre as possíveis causas para a baixa regularidade na realização de citologias oncóticas, assim como para a busca tardia pelo serviço de saúde, o que foi evidenciado pela quantidade de mulheres que não realizou o exame de prevenção (quase a metade das pacientes analisadas). Isso reafirma a importância dos métodos de rastreio regular e do diagnóstico precoce para traçar estadiamentos e definir tratamentos com maiores possibilidades de cura.

As análises mostraram que, de fato, mulheres acima de 40 anos se apresentam estatisticamente em maior número, considerando a amostra analisada de pacientes com incidência de câncer de colo de útero atendidas em apenas uma unidade hospitalar. Isso demonstra a importância de um enfoque das ações e intervenções de políticas públicas nesta faixa etária.

Uma estratégia para lidar com os $25 \%$ de casos analisados que tiveram o desfecho de perda de seguimento, no âmbito hospitalar, seria a criação de um controle ambulatorial interno dos casos diagnosticados de Câncer de Colo Uterino, como, por exemplo, um livro para registros pessoais da paciente (telefone e endereço) e identificação do registro eletrônico (SES), com objetivo de seguimento da patologia e busca ativa, em caso de abandono do tratamento. Outra proposta que favoreceria a completude dos prontuários eletrônicos seria a 
de padronização das consultas com registros de dados relevantes na anamnese, facilitando a compressão dos prontuários e a coleta de dados.

Vale ressaltar que este estudo foi desenvolvido em uma única instituição de saúde no Distrito Federal, sendo necessárias outras pesquisas sobre o tema, para obtenção de perfis epidemiológicos cada vez mais completos e fidedignos, com a finalidade de proporcionar maiores subsídios às políticas públicas de planejamento e obter maior controle da patologia.

\section{REFERÊNCIAS}

1 - Bosch FX, Sanjosé S. Human papillomavirus in cervical cancer. [serial on the Internet] 2002 [cited $2020 \mathrm{dez}$ ]. Available from: link.springer.com/article/10.1007/s11912-002-0079$\mathrm{y}$.

2 - Instituto Nacional De Câncer José Alencar Gomes Da Silva (INCA). Brasil. Ministério da Saúde. Estimativa de câncer no Brasil. Rio de Janeiro: INCA; 2020. [acesso em dez. 2020]. Disponível em: inca.gov.br/numeros-de-cancer.

3 - Brasil. Ministério da Saúde. Diretrizes Brasileiras para o Rastreamento do Câncer de Colo de útero. $2^{\circ}$ ed. Rio de Janeiro. Instituto Nacional do Câncer. 2016 [acesso em dez. 2020]; 118 p. Disponível em: inca.gov.br/sites/ufu.sti.inca.local/files//media/document//diretrizesparaorastreamentodocance rdocolodoutero_2016_corrigido.pdf.

4 - Casarin M, Renata, PJCE. Educação em saúde para prevenção do câncer de colo do útero em mulheres do município de Santo Ângelo/RS. Ciênc. saúde coletiva [Internet]. 2011 Sep [acesso em dez. 2020]; 16 (9): 3925-3932. Disponível em: scielo.br/scielo.php?script=sci_arttext\&pid=S1413-81232011001000029\&lng=en. 
5 - Mccredie, MR.; et al. Natural history of cervical neoplasia and risk of invasive cancer women with cervical intraepithelial neoplasia. 3: a retrospective cohort study. Lancet Oncol. May; 9 (5): 425-34. 2008.

6 - Ferreira MDPL. Prevalência de infecção pelo HPV e seus fatores de risco em adolescentes e mulheres jovens. [Dissertação]. Niterói (RJ): Programa de Pós-Graduação em Ciências Médicas da Universidade Federal Fluminense, 2007 [acesso em dez. 2020]. Disponível em: docplayer.com.br/8391355-Prevalencia-de-infeccao-pelo-hpv-e-seus-fatores-de-risco-emadolescentes-e-mulheres-jovens.html.

7 - Carvalho JP et al. Rastreio, diagnóstico e tratamento do câncer de colo de útero. São Paulo: Federação Brasileira das Associações de Ginecologia e Obstetrícia (FEBRASGO), 2017 [acesso em dez. 2020]. Disponível em: febrasgo.org.br/media/k2/attachments/05ZZDIAGNOYSTICOZRASTREIOZEZTRATAMENTOZDOZCAyNCERZDEZCOLOZDEZ UyTERO.pdf.

8 - Nayar R, Wilbur DC, editors. The Bethesda system for reporting cervical cytology: definitions, criteria, and explanatory notes. 3rd ed. Switzerland: Springer; 2015 [cited 2020 dez]. Available from: fosp.saude.sp.gov.br:443/docs/The+Bethesda+System+for+Reporting+Cervic.pdf. 9 - World Health Organization. National cancer control programmes: policies and managerial guidelines. 2.ed. Geneva: WHO, 2002 [cited 2020 dez]. Available from: apps.who.int/iris/handle/10665/42494.

10 - Ferro Filho AX. Câncer do colo do útero. In: Manual de Ginecologia da Sociedade de Ginecologia e Obstetrícia de Brasília. 2a Ed. Brasília. p. 485. 2017 [acesso em dez. 2020]. Disponível em: www.sgob.org.br/wpcontent/uploads/2017/10/ManualSGOBdigital11102017.pdf. 
11 - Bhatla N, Aoki D, Sharma DN, Sankaranarayanan, R. (2018), Cancer of the cervix uteri. Int J Gynecol Obstet, 143: 22-36. 2019 [cited 2020 dez]. Available from: obgyn.onlinelibrary.wiley.com/doi/epdf/10.1002/ijgo.12611.

12 - Garland SM, et al. Quadrivalent Vaccine Against Human Papillomavirus to Prevent Anogenital Diseases. New England Journal of Medicine; 356: 1928-1943. 2007 [cited 2020 dez]. Available from: nejm.org/doi/full/10.1056/nejmoa061760.

13 - Brasil. Ministério da Saúde. Secretaria de Vigilância em Saúde. Departamento de Vigilância das Doenças Transmissíveis. Manual de Normas e Procedimentos para Vacinação / Ministério da Saúde, Secretaria de Vigilância em Saúde, Departamento de Vigilância das Doenças Transmissíveis. - Brasília: Ministério da Saúde, 2014.176 p.: il. ISBN 978-85-3342164-6. 2014 [acesso em dez. 2020]. Disponível em: bvsms.saude.gov.br/bvs/publicacoes/manual_procedimentos_vacinacao.pdf.

14 - Gonçalves AL, et al. Série de casos de mulheres com câncer de colo uterino em hospital público de Brasília. Braz. J. Hea. Rev., Curitiba, v. 3, n. 3, p.3920-3934 may./jun. ISSN 25956825. 2020 [acesso em dez. 2020]. Disponível em: brazilianjournals.com/index.php/BJHR/article/view/9468.

15 - Bray F, et al. Global cancer statistics 2018: GLOBOCAN estimates of incidence and mortality worldwide for 36 cancers in 185 countries. CA Cancer J Clin; 68(6): 394-424. DOI: 10.3322/caac.21492. 2018 [cited 2020 dez]. Available from: acsjournals.onlinelibrary.wiley.com/doi/full/10.3322/caac.21492.

16 - Brasil. Ministério da Saúde. Instituto Nacional De Câncer José Alencar Gomes Da Silva (INCA). Estimativa 2020: Síntese de Resultados e Comentários. Rio de Janeiro: INCA; 2020. [acesso em fev. 2021]. Disponível em: inca.gov.br/estimativa/sintese-de-resultados-ecomentarios. 
17 - Brasil. Ministério da Saúde. Instituto Nacional De Câncer José Alencar Gomes Da Silva (INCA). Conceito e Magnitude. Rio de Janeiro: INCA; 2020. [acesso em dez. 2020]. Disponível em: inca.gov.br/controle-do-cancer-do-colo-do-utero/conceito-e-magnitude. 